Angiocentric features are uncommon in high-grade World Health Organisation (WHO) brain tumours, whilst they are typical for WHO grade I tumours, e.g. angiocentric gliomas. We present an unusual glial tumour that occurred in a 59-year-old man. The tumour had equivocal radiologic and histopathologic features, especially a characteristic angiocentric pattern, low-to-moderate Ki67, and dot-like epithelial membrane antigen expression. The tumour did not show features characteristic for glioblastoma; however, it recurred as glioblastoma four months later. Based on this case, we show that high-grade WHO brain tumours may show an angiocentric pattern typical for low-grade WHO brain tumours, such as angiocentric gliomas.

Key words: angiocentric glioma, glioblastoma, anaplastic astrocytoma, ependymal differentiation, angiocentric features.

Contemp Oncol (Pozn) 2018; 22 (3): 205-208 DOI: https://doi.org/10.5114/wo.2018.78944

\section{A malignant astrocytoma with uncommon angiocentric features and dot-like EMA expression}

\author{
Wojciech Kuncman ${ }^{1 \star}$, Marcin Braun ${ }^{1 \star}$, tukasz Kuncman ${ }^{2}$, Piotr Kupnicki ${ }^{3}$, \\ Michał Piotrowski ${ }^{4}$, Dorota Jesionek-Kupnicka' ${ }^{1}$, Radzisław Kordek ${ }^{1}$
}

${ }^{1}$ Department of Pathology, Chair of Oncology, Medical University of Lodz, Poland ${ }^{2}$ Department of Radiotherapy, Chair of Oncology, Medical University of Lodz, Poland ${ }^{3}$ Department of Radiology and Diagnostic Imaging, Medical University of Lodz, Poland ${ }^{4} \mathrm{Clinic}$ of Neurosurgery and Oncology of the Nervous System, Medical University of Lodz, Poland

*These authors contributed equally to this work as first authors.

\section{Introduction}

Angiocentric features are uncommon in high-grade WHO brain tumours and are more suggestive of low-grade WHO tumours, e.g. angiocentric gliomas (AGs) [1-3]. AGs are epilepsy-associated, benign neuroepithelial tumours that occur typically among children and young adults, and only rarely in adult patients [2, 3]. AGs grow slowly and display characteristic angiocentric structures composed of bipolar, spindled cells with no atypia and ependymal differentiation [2-5]. AGs are considered curable with surgery; however, there were seven tumour recurrences from approximately one hundred tumours with angiocentric pattern described in the literature [4].

We present an unusual malignant glial tumour with prominent angiocentric features, low Ki-67, and dot-like EMA expression, which occurred in a 59-year-old patient.

\section{Case report}

In April 2014, a 59-year-old man was admitted to the Emergency Department with tonic-clonic seizures. No brain tumour was revealed in contrast-enhanced computed tomography (CT). In December, dysphasia and sensory disturbances of the right upper limb were observed, and a second CT scan was taken. It revealed a small, heterogenously hyperdense haematoma surrounded by oedema. The initial radiologic diagnosis was haemorrhagic stroke. However, in subsequent magnetic resonance imaging (MRI) a tumour in the rear part of parietal lobe was shown (Fig. 1A-1B). The coronal T1 WI showed a peripheral irregular ring enhancement around the area resembling necrosis. The T2 WI showed that the tumour was surrounded by a vasogenic oedema. The patient underwent a subtotal cranial tumourectomy; however, only a small part of the tumour was recovered for histopathological examination (Fig. 2A-2B).

In the histopathologic examination, the tumour was mainly composed of spindle pleomorphic cells with no mitotic activity. These cells formed characteristic pseudorosettes arranged around blood vessels. A wide variety of cellularity with areas of palisading schwannoma-like pattern was observed. The Ki-67/MIB-1 labelling index was low - up to 5\% (Fig. 3B). These features vouched for the diagnosis of angiocentric glioma (Fig. 3A); however, small foci of higher cellularity were found. In these areas, the tumour cells presented distinct nuclear atypia and a higher Ki-67/MIB1 labelling index of $6 \%$ to $8 \%$, which indicated on higher grade (Fig. 3C). Necrosis and microvascular proliferations were absent; however, the material from the surgery might have been underrepresented. Staining for epithelial membrane anti- 


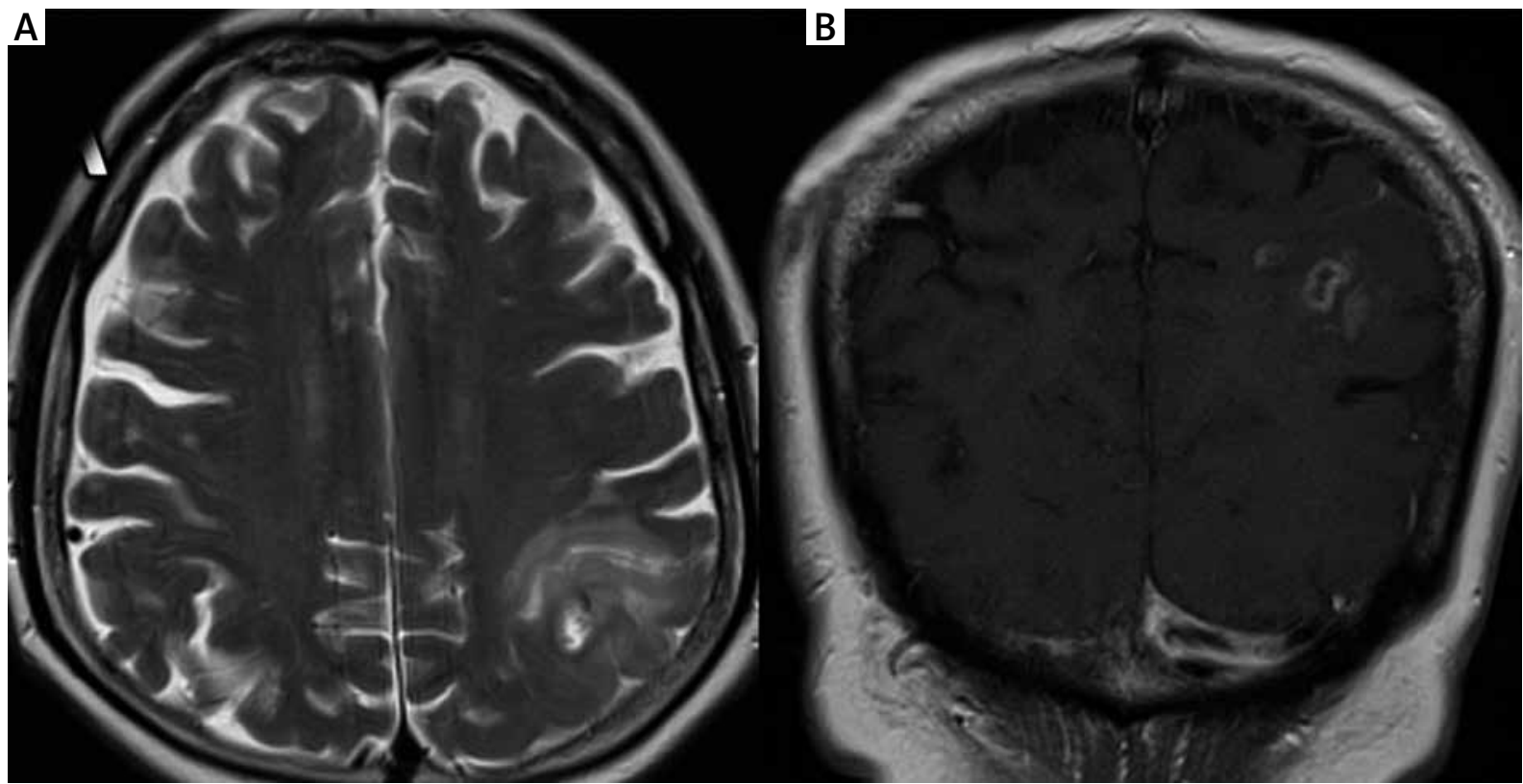

Fig. 1. The MRI of the first tumour suggestive for a high-grade glioma. A) T2WI - the tumour is surrounded by vasogenic oedema. B) T1 WI - a ring enhancement around an area resembling necrosis with peripheral irregular enhancement

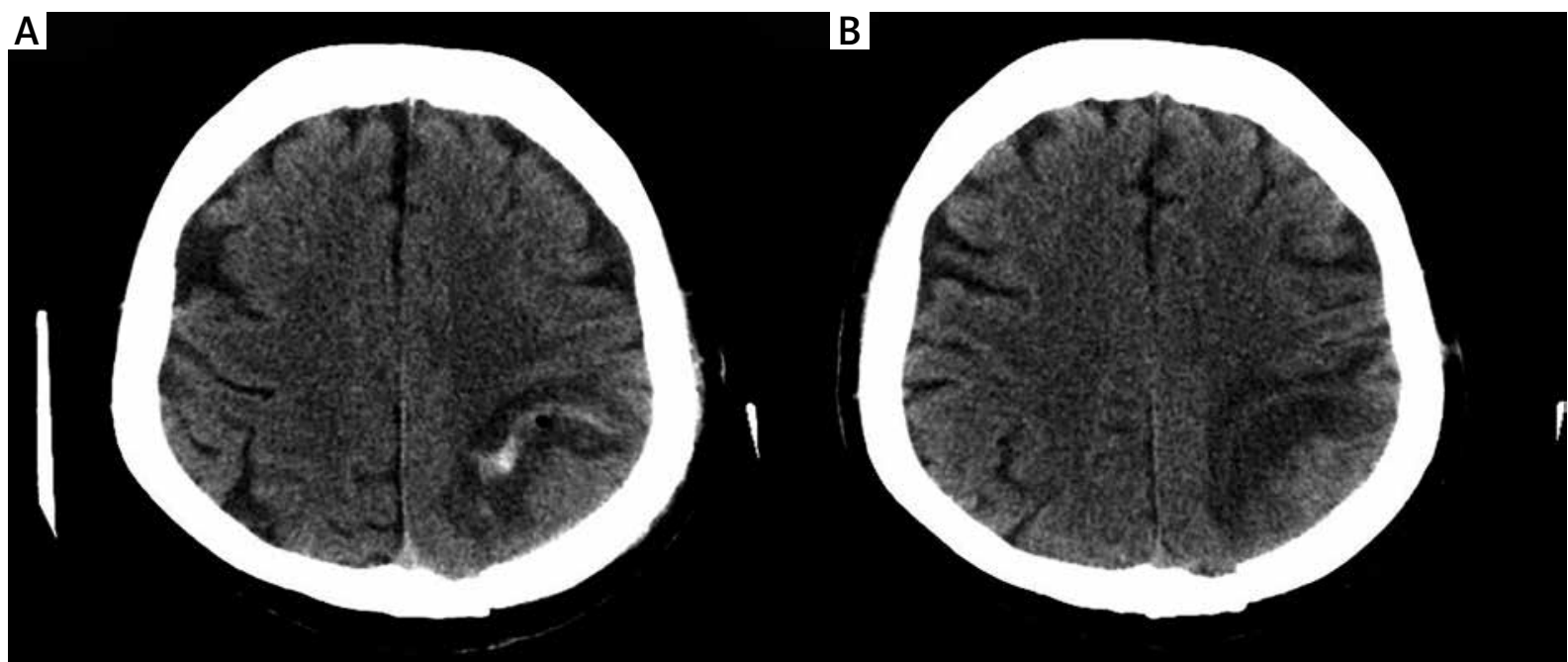

Fig. 2. The control CT images after the first tumourectomy. A) After two weeks. An oedema with haematoma along with air bubbles can be seen. B) After two months. The residuals of the tumour can be seen

gen (EMA), which is a marker for epithelial differentiation, revealed two different patterns. The first was diffuse and the second was dot-like and highlighted microlumens (Fig. 3D). Immunohistochemical staining for glial fibrillary acidic protein (GFAP), epidermal growth factor receptor (EGFR), podoplanin (D2-40), S-100, and vimentin were positive, whilst stainings for synaptophysin, $\mathrm{R} 132 \mathrm{H}$-mutated isocitrate dehydrogenase 1 (IDH1), p16, and p53 were negative. Considering the unusual histology and the CT/MRI presentation, we suggested a diagniosis of malignant astrocytoma with angiocentric features.

The patient remained in a good condition for the next four months, but then he presented with partial paralysis of the right upper limb and speech disorders. The MRI showed a recurrent tumour with central necrosis surrounded by oedema with peripheral and irregular enhancement.
The patient was reoperated and sufficient material was obtained for the histopathological examination.

On histology, the lesion presented with a heterogeneous structure. There were fields with characteristic angiocentric features that corresponded to those of the primary tumour, but the neoplasm lost the expression of EMA (Fig. 3F). Additionally, there were unequivocal glioblastoma areas with necrosis and microvascular proliferations (Fig. 3G-3H). A few mitoses and multinuclear tumour cells were also found. The Ki-67/MIB-1 index was up to 30\% in hot spots. The stains for GFAP, vimentin, and p53 were strong. Interestingly, S100 and D2-40 stains were inhomogeneous with no expression in the most poorly differentiated areas. Broad-spectrum cytokeratins were weakly positive only in small spots.

Following surgery, the patient presented with paresis of the right upper limb. Subsequently, he was qualified to 


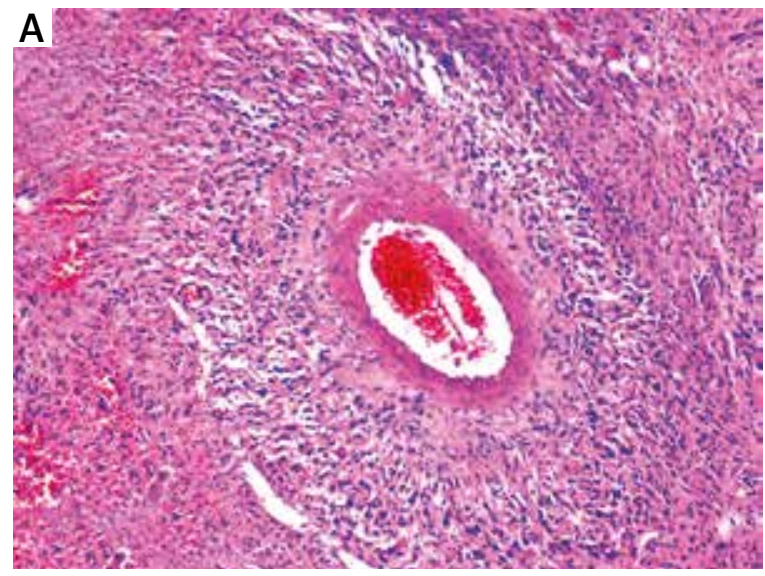

B ogating

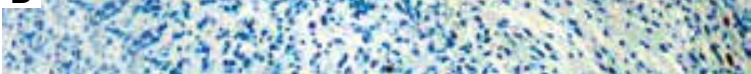

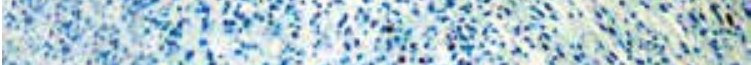

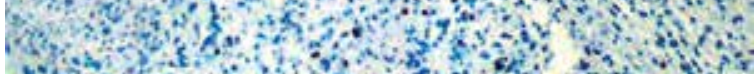

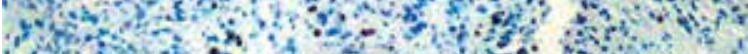

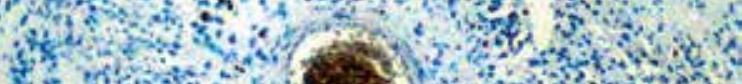

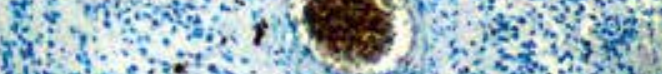

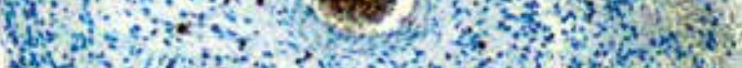

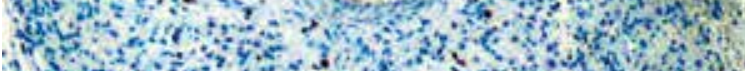

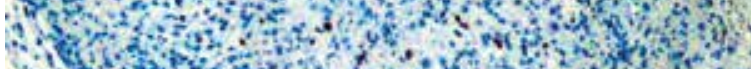

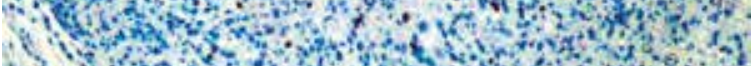
A. Q

C 5 the

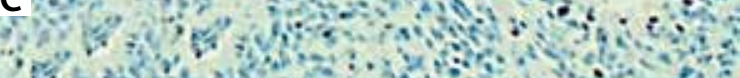

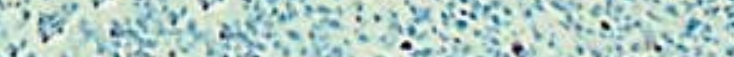

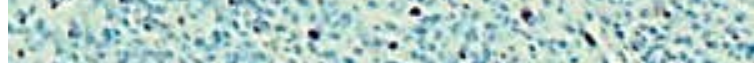

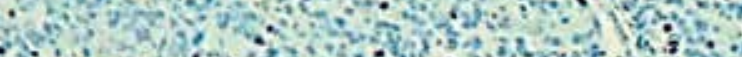

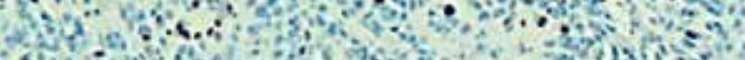

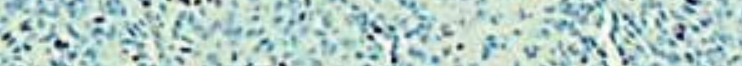

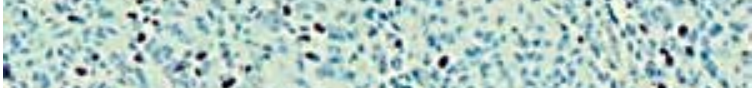
a d s d

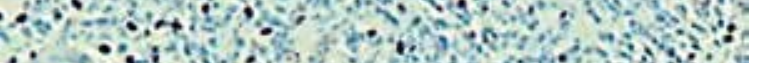

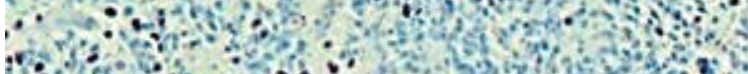

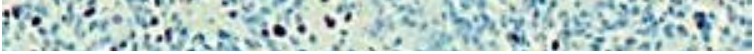

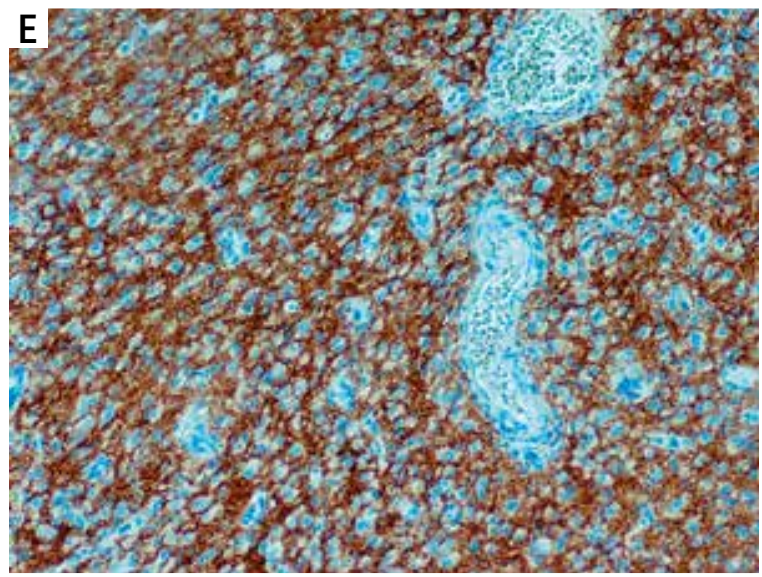

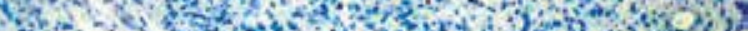
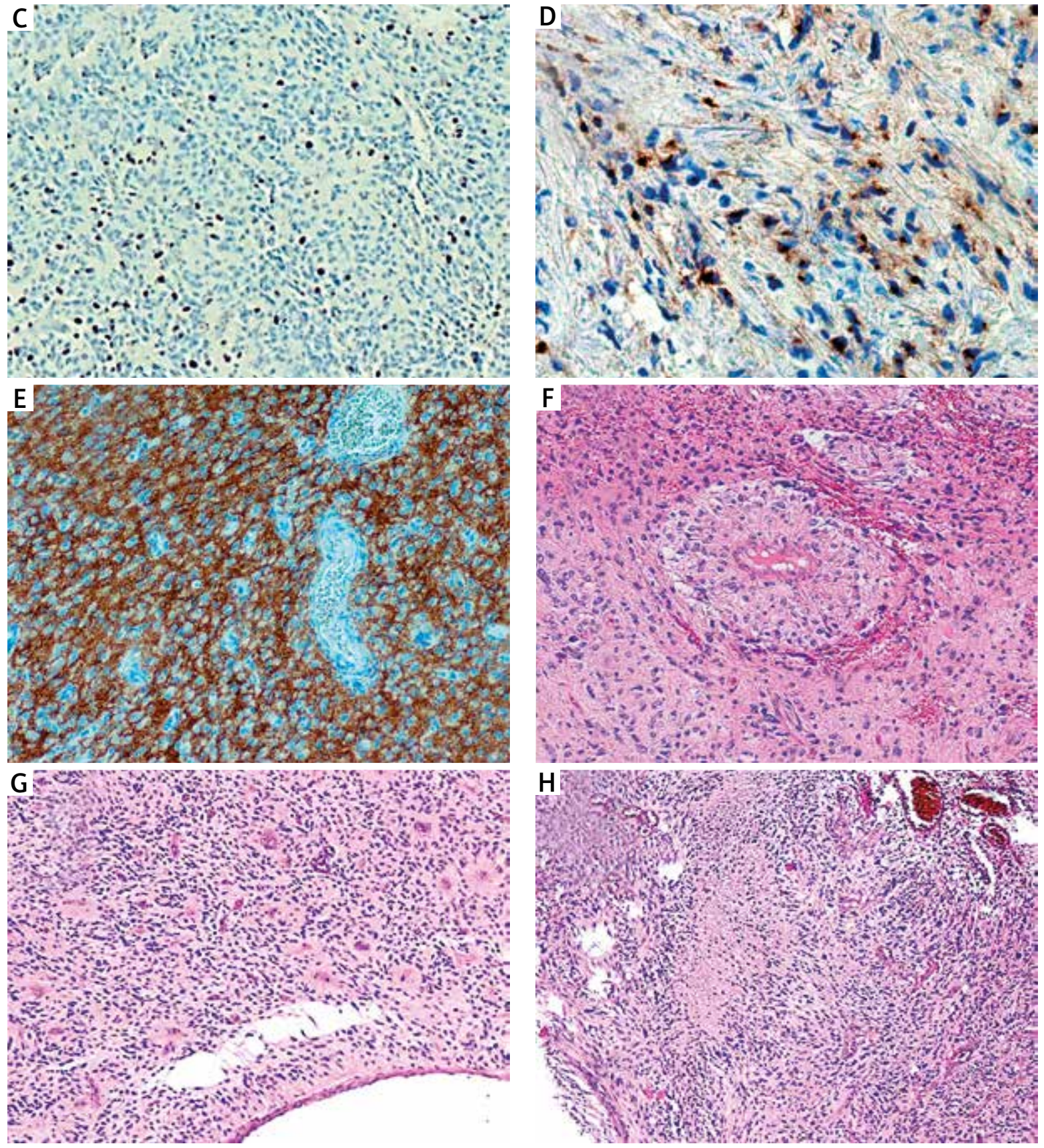

Fig. 3. Images of primary (A-E) and recurrent (F-H) tumours. A) Angiocentric pattern in H\&E. B) Ki-67 index below 5\%. C) Small areas with Ki67 index of 5-10\%. D) Dot-like epithelial membrane antigen (EMA). E) Diffuse EMA staining. F) Reaming angiocentric pattern. G) Microvascular proliferations and cellular atypia. $\mathrm{H}$ ) Focal necrosis and residual angiocentric pattern 
combined radio- and chemotherapy with temozolomide. The treatment tolerance was satisfactory. A control MRI after a second cycle revealed recurrence, and a salvage surgery was performed. Histopathological examination confirmed the diagnosis of glioblastoma. The patient died two weeks after the third surgery and 11 months after the first resection.

\section{Discussion}

We present an unusual astrocytoma with prominent angiocentric features, which recurred rapidly as an unequivocal glioblastoma. Our case shows that angiocentric features and dot-like EMA expression should not be considered as pathognomonic for angiocentric glioma and may be present in high-grade WHO astrocytomas.

On the one hand, the tumour presented with angiocentric areas, which were composed of bipolar cells of moderate atypia, and low Ki-67 index and expression of EMA. There was no microvascular proliferation and no necrosis. The immunohistochemical examination showed dot-like EMA expression and regular cytoplasmic expression of podoplanin (D2-40), both characteristic for ependymal differentiation [1-3]. The tumour was negative for expression of R132H-mutant IDH1, which vouched against WHO grade II or III astrocytoma [3]. On the first presentation, many microscopic findings suggested a benign nature of the lesion and corresponded with angiocentric glioma (WHO grade I) $[2,3]$.

Conversely, the primary tumour had small areas of higher cellularity with nuclear atypia and higher Ki67 index, which suggested astrocytoma of higher grade. The age of the patient was not characteristic for AG [2-5]. Finally, although the radiologic presentation was equivocal, it was suggestive of a high-grade glioma (Fig. 1A-1B). Considering all aspects, we diagnosed the primary tumour as being suggestive of high-grade astrocytoma with angiocentric features probably arising from a low-grade astrocytoma. However, we could not exclude that the material was underrepresented in the first histopathological examination, and a true high-grade component was just not recovered for histopathology. Unfortunately, the problem with small resections is common in the diagnosis and management of brain tumours [2, 3]. Our diagnostic problems also show the value of a detailed radiological examination for the neuropathologist. Our patient progressed five months after surgery into a glioblastoma-preserving angiocentric component. This finally confirmed the malignant nature of the primary tumour, but the concerns during diagnosis raise the question of whether the tumour was primarily an anaplastic astrocytoma with angiocentric features or a glioblastoma with a lower-grade component with angiocentric features.

There are seven tumour recurrences from approximately 100 tumours with angiocentric pattern described in the literature [2-12]. Most of them had atypical histopathological findings like pleomorphism, mitotic activity, or high Ki-67 index and angiocentric pattern with ependymal differentiation. Three cases progressed to high-grade glioma without AG features $[4,7,8,11]$. Two cases of high-grade gliomas with angiocentric features have been described
$[7,8]$. These few cases, including ours, raise the question of whether high-grade astrocytomas can mimic angiocentric glioma or if the progression of early undetected angiocentric glioma exists. We agree with the suggestions of Miyahara et al. that it would be reasonable to classify a new subtype of glial tumours as "anaplastic astrocytoma with angiocentric ependymal differentiation” [8].

The authors declare no conflict of interest.

\section{References}

1. Lehman NL. Central nervous system tumours with ependymal features: a broadened spectrum of primary ependymal differentiation? J Neuropathol Exp Neurol 2008; 67: 177-188.

2. Louis DN, Ohgaki H, Wiestler OD, Cavenee WK, Burger PC, Jouvet A, Scheithauer BW, Kleihues P. The 2007 WHO classification of tumours of the central nervous system. Acta Neuropathol 2007; 114: 97-109.

3. Louis DN, Perry A, Reifenberger G, et al. The 2016 World Health Organization Classification of Tumors of the Central Nervous System: a summary. Acta Neuropathol 2016; 131: 803.

4. Ampie L, Choy W, DiDomenico JD, Lamano JB, Williams CK, Kesavabhotla K, Mao Q, Bloch O. Clinical attributes and surgical outcomes of angiocentric gliomas. J Clin Neurosci 2016; 110: 375-380.

5. Grajkowska W, Matyja E, Daszkiewicz P, Roszkowski M, Peregud-Pogorzelski J, Jurkiewicz E. Angiocentric glioma: a rare intractable epilepsy-related tumour in children. Folia Neuropathol 2014; 52: 253-259.

6. Liu CQ, Zhou J, Qi X, Luan GM. Refractory temporal lobe epilepsy caused by angiocentric glioma complicated with focal cortical dysplasia: a surgical case series. J Neurooncol 2012; 110: 375-380.

7. Lu JQ, Patel S, Wilson BA, Pugh J, Mehta V. Malignant glioma with angiocentric features. J Neurosurg Pediatr 2013; 11: 350-355.

8. Miyahara H, Toyoshima Y, Natsumeda M, et al. Anaplastic astrocytoma with angiocentric ependymal differentiation. Neuropathology 2011; 31: 292-298.

9. Ni HC, Chen SY, Chen L, Lu DH, Fu YJ, Piao YS. Angiocentric glioma: a report of nine new cases, including four with atypical histological features. Neuropathol Appl Neurobiol 2015; 41: 333-346.

10. Sugita Y, Ono T, Ohshima K, Niino D, Ito M, Toda K, Baba H. Brain surface spindle cell glioma in a patient with medically intractable partial epilepsy: A variant of monomorphous angiocentric glioma? Neuropathology 2008; 28: 516-520.

11. Wang M, Tihan T, Rojiani AM, et al. Monomorphous angiocentric glioma: a distinctive epileptogenic neoplasm with features of infiltrating astrocytoma and ependymoma. J Neuropathol Exp Neurol 2005; 64: 875-881.

12. Miyata H, Ryufuku M, Kubota Y, Ochiai T, Niimura K, Hori T. Adult-onset angiocentric glioma of epithelioid cell-predominant type of the mesial temporal lobe suggestive of a rare but distinct clinicopathological subset within a spectrum of angiocentric cortical ependymal tumours. Neuropathology 2012; 32: 479-491.

\section{Address for correspondence}

\section{Marcin Braun}

Department of Pathology, Chair of Oncology

Medical University of Lodz

251 Pomorska St.

92-213 Lodz, Poland

e-mail: braunmarcin@gmail.com

Submitted: 18.06 .2018

Accepted: $\quad 15.08 .2018$ 